\title{
Electrical Conductivity and Magnetic Susceptibility of Thallium 1223-Based Superconductor in the Fluctuation Range
}

\author{
W. M. Woch ${ }^{1} \cdot$ R. Zalecki ${ }^{1} \cdot$ M. Giebułtowski ${ }^{1} \cdot$ M. Kowalik ${ }^{1} \cdot$ J. Niewolski ${ }^{1} \cdot$ J. Przewoźnik $^{1}$ \\ Received: 6 March 2018 / Accepted: 16 April 2018 / Published online: 4 May 2018 \\ (C) The Author(s) 2018
}

\begin{abstract}
The paper reports on the temperature dependencies of the electrical resistivity and the a.c. magnetic susceptibility of the $\mathrm{Tl}_{0.8} \mathrm{Bi}_{0.3} \mathrm{Sr}_{1.8} \mathrm{Ba}_{0.2} \mathrm{Ca}_{2} \mathrm{Cu}_{3} \mathrm{O}_{x}$ bulk superconductor measured in the area of the transition from the metallic to the superconducting state. From the resistivity data, the onset temperature $T_{\mathrm{c} \text {, onset }}=121.6 \mathrm{~K}$, the critical temperature $T_{\mathrm{c} 50 \%}=118.8 \mathrm{~K}$, the zero resistance critical temperature obtained using $E=10^{-6} \mathrm{~V} / \mathrm{cm}$ criterion $T_{\mathrm{c} 0}=117.9 \mathrm{~K}$, and the width of superconducting transition $\Delta T=1.6 \mathrm{~K}$ were derived. The thermal fluctuations were examined using the formulae for the conductance: $\Delta \sigma=K \varepsilon^{-\lambda}$, and for the magnetic susceptibility: $\chi=C \varepsilon^{-\lambda}$, where $\Delta \sigma=1 / R(T)-1 / R_{R}(T), \varepsilon=$ । $\left(T-T_{\mathrm{c}}\right) / T_{\mathrm{c}} \mathrm{l}, \lambda$ is the critical exponent, and $K$ and $C$ are constants. The critical exponents in the transition region from the normal to the superconducting state were determined and analyzed. The three-dimensional fluctuations were found to occur in the vicinity of the zero-resistance critical temperature.
\end{abstract}

Keywords Thallium-based superconductors $\cdot$ Electrical conductivity $\cdot$ Magnetic susceptibility $\cdot$ Fluctuation phenomena

\section{Introduction}

Phase transitions, which include the transition from the metallic to the superconducting state, are accompanied by critical phenomena which are characterized by singularities of physical quantities where they go to zero or diverge. This is a direct consequence of the correlation length $\xi$ diverging and becoming larger than every other physical length scale, so the divergence of $\xi$ dominates the physics. In particular, thermal fluctuations (thermodynamic) occur near the critical temperature. If the entropy dominates the Ginzburg-Landau free energy, as the temperature rises approaching the critical temperature, the fluctuations proliferate. When the temperature is higher than the critical temperature, i.e., in the normal state of the superconductor, superconducting islands with sizes of several coherence lengths appear. As we approach the transition temperature, the superconducting islands grow significantly and connect with each other so that the

W. M. Woch

wmwoch@agh.edu.pl

1 Department of Solid State Physics, Faculty of Physics and Applied Computer Science, AGH University of Science and Technology, Ave. Mickiewicza 30, 30-059 Cracow, Poland normal areas become islands. When we approach the critical temperature from above, normal areas become small islands. A large penetration depth $\lambda$ of $10^{3}-10^{4} \AA$ [1], small coherence length $\xi$ of $10 \AA$ [2], high anisotropy $[3,4]$ as well as high critical temperatures considerably enhance the role of thermal fluctuations in high-temperature superconductors (HTS). After the discovery of HTS, papers treating thermal fluctuation problems appeared, in which the excess conductivity in the transition ranges for yttrium- [5-7], bismuth- [8, 9], and thallium [10-12]-based superconductors was most often studied.

In the critical region, there occur two types of fluctuations: critical fluctuations at temperatures close to the critical temperature and stochastic (Gaussian) fluctuations above it. It has been shown that thermal fluctuations depend on the applied magnetic field and the applied pressure [1315]. Also, two Gaussian regions were observed, one of them being localized in the vicinity of the zero-resistance critical temperature [16].

In this paper, we present and discuss a study of critical exponents in the fluctuation region of a ceramic $\mathrm{Tl}_{0.8} \mathrm{Bi}_{0.3} \mathrm{Sr}_{1.8} \mathrm{Ba}_{0.2} \mathrm{Ca}_{2} \mathrm{Cu}_{3} \mathrm{O}_{x}$ superconductor derived from the electrical resistance and the a.c. magnetic susceptibility measurements. We utilize the fact that electrical resistance measurements allow us to study thermal fluctuations above critical temperature while magnetic susceptibility 
measurements allow us to investigate them below critical temperature. This provides a very interesting look at critical exponents across the whole range where fluctuations play a significant role.

\section{Experimental}

The sample of the ceramic thallium-based $\mathrm{Tl}_{0.8} \mathrm{Bi}_{0.3} \mathrm{Sr}_{1.8} \mathrm{Ba}_{0.2}$ $\mathrm{Ca}_{2} \mathrm{Cu}_{3} \mathrm{O}_{x}$ (1223) superconductor, partly substituted with $\mathrm{Bi}$ for $\mathrm{Tl}$ and with $\mathrm{Ba}$ for $\mathrm{Sr}$, was prepared in two stages using a standard solid-state reaction technique presented in the literature [17]. At the first stage, the thallium-free precursor was obtained from the appropriate amounts of $\mathrm{CuO}, \mathrm{Bi}_{2} \mathrm{O}_{3}, \mathrm{SrCO}_{3}, \mathrm{BaCO}_{3}$, and $\mathrm{CaCO}_{3}$. The starting compounds were thoroughly mixed and ground into a fine powder. Next the powder was calcined at the temperature $880{ }^{\circ} \mathrm{C}$ for $20 \mathrm{~h}$ in air and then reground into a fine powder. At the second stage, the amounts of $\mathrm{Tl}_{2} \mathrm{O}_{3}$ and $\mathrm{Bi}_{2} \mathrm{O}_{3}$ appropriate for the starting composition $\mathrm{Tl}_{0.8} \mathrm{Bi}_{0.3} \mathrm{Sr}_{1.8} \mathrm{Ba}_{0.2} \mathrm{Ca}_{2} \mathrm{Cu}_{3} \mathrm{O}_{x}$ were added to the calcined precursor powder and thoroughly mixed for $1 \mathrm{~h}$. Then, the powder was pressed into a pellet at the pressure of $0.6 \mathrm{GPa}$. The pellet, wrapped in a silver foil, was then sintered at the temperature of $910{ }^{\circ} \mathrm{C}$ for $20 \mathrm{~h}$ in the atmosphere of flowing oxygen. Heating rates were $3 \mathrm{~K} / \mathrm{min}$ up to $850{ }^{\circ} \mathrm{C}$ and $1 \mathrm{~K} / \mathrm{min}$ up to $910{ }^{\circ} \mathrm{C}$. Then, the sample was cooled down to room temperature at the rate of $1 \mathrm{~K} / \mathrm{min}$. To obtain a good homogeneity, the pellet was reground, milled, repressed, and resintered at the same conditions. The pellet was subsequently unwrapped from the silver foil and annealed at $740{ }^{\circ} \mathrm{C}$ for $15 \mathrm{~h}$ in the atmosphere of flowing oxygen. Then, it was cooled down to room temperature at the rate of $1 \mathrm{~K} / \mathrm{min}$, as before. From the pellet, a cuboidal sample was cut out with the dimensions of $4.0 \times 4.7 \times 0.25 \mathrm{~mm}^{3}$.

The measurement of the X-ray diffraction (XRD) of the Tl-1223 sample was carried out with the Empyrean Panalytical diffractometer $(\mathrm{CuK} \alpha$ radiation) at $300 \mathrm{~K}$ in the $2 \theta$ range from 2 to $100^{\circ}$. The electrical resistance versus temperature was measured using the four-probe a.c. method. The electrical contacts on the sample were made by Leitsilber 200 silver paint, and their resistance after curing at $300{ }^{\circ} \mathrm{C}$ was less than $0.8 \mathrm{Ohm}$. The dispersion $\chi^{\prime}$ and absorption $\chi^{\prime \prime}$ parts of the a.c. susceptibility as a function of temperature at the magnetic field amplitude of 20 mOe were measured by a standard mutual inductance bridge operating at the frequency of $189 \mathrm{~Hz}$. A Stanford SR 830 lock-in nanovoltmeter served both as a source of the a.c. current for the primary coil producing the a.c. magnetic field and as a voltmeter of the bridge. The temperature was controlled with the accuracy of $\pm 0.05 \mathrm{~K}$ by a Lake Shore temperature controller employing a chromel-gold- $0.07 \% \mathrm{Fe}$ thermocouple for both experimental setups.

\section{Results and Discussion}

The X-ray diffraction pattern of the ceramic $\mathrm{Tl}_{0.8} \mathrm{Bi}_{0.3} \mathrm{Sr}_{1.8}$ $\mathrm{Ba}_{0.2} \mathrm{Ca}_{2} \mathrm{Cu}_{3} \mathrm{O}_{x}$ is shown in Fig. 1. The diffraction spectrum was analyzed using FULLPROF software based on the Rietveld method [18]. Analysis of the XRD patterns showed that the sample contains two superconducting phases: the majority 1223 superconducting phase with the critical temperature $T_{\mathrm{c}, 50 \%}=118.8 \mathrm{~K}$ as determined from resistance measurement and a minority 1212 superconducting phase that was not observed in either resistance or susceptibility measurements and which critical temperature reported in the papers $[19,20]$ is of $100 \mathrm{~K}$. The sample was found to contain also small amounts of non-conducting phases: $\mathrm{CaCu}_{2} \mathrm{O}_{3}, \mathrm{CaO}, \mathrm{SrBiO}_{3}$, and $\mathrm{Sr}_{8} \mathrm{Ca}_{6} \mathrm{Cu}_{24} \mathrm{O}_{41}$.

The temperature dependence of the resistance is shown in Fig. 2. From this measurement, the following values of critical temperatures are obtained: the onset temperature $T_{\mathrm{c} \text {,onset }}=121.6 \mathrm{~K}$ and the critical temperature $T_{\text {c50\% }}=118.8 \mathrm{~K}$. The zero resistance critical temperature determined with the $E=10^{-6} \mathrm{~V} / \mathrm{cm}$ criterion (see inset in Fig. 2) equals $T_{\mathrm{c} 0}=117.9 \mathrm{~K}$, and the width of superconducting transition amounts to $\Delta T=T_{\mathrm{c} 90 \%}-$ $T_{\mathrm{c} 10 \%}=1.6 \mathrm{~K}$.

An important issue associated with thermal fluctuations is the problem of the dimensionality of superconductivity. The classical theory of fluctuations [21, 22] predicts that in three-dimensional superconductivity, the excess conductivity can be written as

$\Delta \sigma \propto\left(\frac{T-T_{\mathrm{c}}}{T_{\mathrm{c}}}\right)^{-\lambda}$

with the critical exponent $\lambda=0.5$.

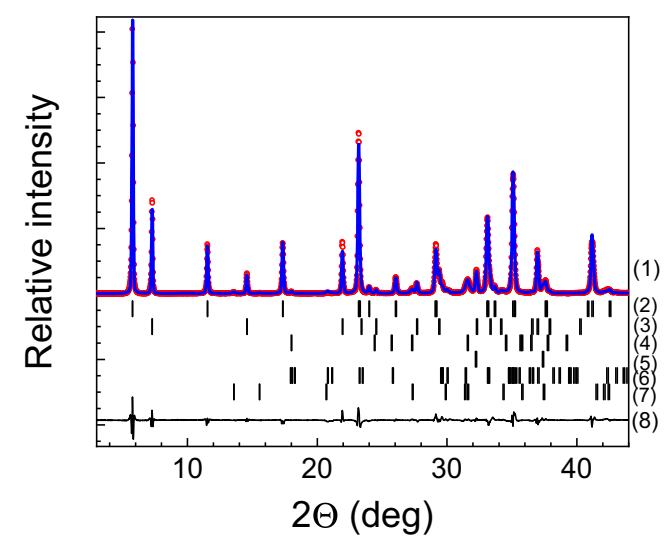

Fig. $1 \mathrm{X}$-ray diffraction pattern of the $\mathrm{Tl}_{0.8} \mathrm{Bi}_{0.3} \mathrm{Sr}_{1.8} \mathrm{Ba}_{0.2} \mathrm{Ca}_{2} \mathrm{Cu}_{3} \mathrm{O}_{x}$ sample with $\mathrm{CuK} \alpha$ radiation. (1) experimental data (open circle), Rietveld's analysis (solid line); (2) Tl-1223 phase; (3) Tl-1212 phase; (4) $\mathrm{CaCu}_{2} \mathrm{O}_{3}$ compound; (5) $\mathrm{CaO}$ compound; (5) $\mathrm{SrBiO}_{3}$ compound; (6) $\mathrm{Sr}_{8} \mathrm{Ca}_{6} \mathrm{Cu}_{24} \mathrm{O}_{41}$ compound; (7) differences 


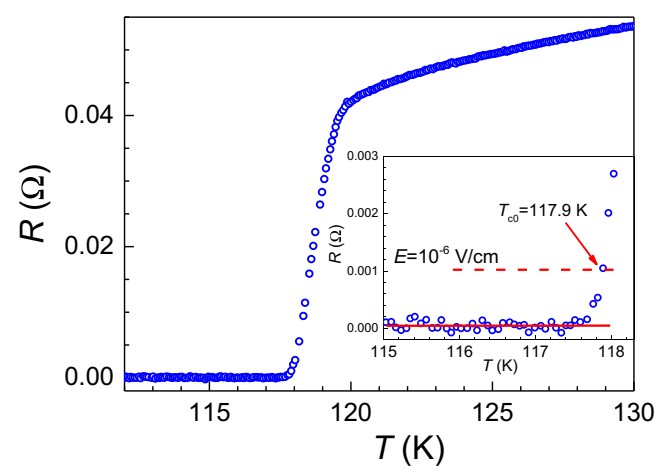

Fig. 2 Temperature dependence of the resistance of the $\mathrm{Tl}_{0.8} \mathrm{Bi}_{0.3} \mathrm{Sr}_{1.8} \mathrm{Ba}_{0.2} \mathrm{Ca}_{2} \mathrm{Cu}_{3} \mathrm{O}_{x}$ (1223) bulk superconductor. Inset: illustration of the way of the zero critical temperature determination using the $E=10^{-6} \mathrm{~V} / \mathrm{cm}$ criterion

Due to the large anisotropy, as well as the exceptional broadening of the superconducting transition, HTS materials are described by a three-dimensional (3D) to twodimensional (2D) transition in the critical region. This concerns the ceramics, single crystals as well as the thin film samples [23-26]. A crossover from 2D to $1 \mathrm{D}$ was also reported for Hg-Tl 1223 ceramic superconductor [27]. The excess conductivity of the lower-dimensionality superconductors could successfully be described by the theory [28], which predicts $\lambda=1$ for the two-dimensional and $\lambda=1.5$ for the one-dimensional case in (1).

When the temperature is approaching the critical temperature, the electrical conductance, magnetic susceptibility, specific heat, and other properties exhibit temperature behavior which can be described by critical exponents. Their values obtained from the experiments can be used for a study of the superconducting fluctuations within the critical region.

At the first stage, the critical exponents determined from experimental data of the electrical resistance are considered. The data have been analyzed within a simple model in which the thermal fluctuations of the conductivity vary according to a power law described by the formula:

$\Delta \sigma=K \varepsilon^{-\lambda}$,

where $\varepsilon=\left|\frac{T-T_{\mathrm{c}}}{T_{\mathrm{c}}}\right|, T_{\mathrm{c}}$ is the critical temperature, $\lambda$ is the critical exponent, and $K$ is a constant. The temperature dependence of the excess conductivity is defined within the Ginzburg-Landau mean field approximation [29] as

$\Delta \sigma(T)=\frac{1}{R(T)}-\frac{1}{R_{R}(T)}$,

where $R(T)$ is the resistivity measured and $R_{R}(T)$ is the resistivity obtained by a linear extrapolation of the experimental data from about $170 \mathrm{~K}$ down to the temperature of the onset of the transition. The determination of $\Delta \sigma$ involves the evaluation of $R_{R}$ for temperatures close to $T_{\mathrm{c}}$ by extrapolation from a higher temperature in the following way:

$R_{R}(T)=R_{0}+\left(\frac{\mathrm{d} R}{\mathrm{~d} T}\right) T$,

where $R_{0}$ and $\mathrm{d} R / \mathrm{d} T$ are constants. This method of determination of critical exponents was described in the paper [30]. The critical exponent $\lambda$ is usually obtained from (2) after the transformation to the logarithmic formula:

$\log \Delta \sigma=-\lambda \log \varepsilon+$ const,

where the critical exponent $\lambda$ is equal to the negative value of the slope of the line fitted to the linear part of this dependence. The function $\log \Delta \sigma$ substantially depends on the definition of the critical temperature appearing in (2). The linear parts of the dependence expressed by (5) were used to derive the critical exponents.

The critical temperature $T_{\mathrm{c}}$ is well defined for the superconductors which have narrow transitions to the superconducting state. In high-temperature superconductors, however, the definition of $T_{\mathrm{c}}$ is more complicated because of the broadening of the transition. In general, this critical temperature is expected to be in the vicinity of the zero resistance critical temperature, to include all the fluctuations appearing within the transition. In the papers [3, 16, 31], the authors accept the temperature for which the derivative $\mathrm{d} R / \mathrm{d} T$ reaches its maximum as a critical temperature and usually denote it as $T_{\mathrm{cp}}$, and substitute in the argument $\varepsilon$ in (2). The temperature dependence of the first derivative of the resistance of the $\mathrm{Tl}_{0.8} \mathrm{Bi}_{0.3} \mathrm{Sr}_{1.8} \mathrm{Ba}_{0.2} \mathrm{Ca}_{2} \mathrm{Cu}_{3} \mathrm{O}_{x}$ (1223) bulk superconductor and its fit with a single Gauss function is shown in Fig. 3. The peak position corresponds to $T_{\mathrm{cp}}=118.7 \mathrm{~K}$ and is very close to $T_{\mathrm{c}, 50 \%}$ critical temperature for this sample. The temperature $T_{\mathrm{cp}}$ is located in the middle of the resistive transition. From this point of view, the zero resistance temperature, $T_{\mathrm{c} 0}=117.9 \mathrm{~K}$, should be taken as the critical temperature in the argument $\varepsilon$ to study whole fluctuations region. The relation corresponding to (5) for the $\mathrm{Tl}_{0.8} \mathrm{Bi}_{0.3} \mathrm{Sr}_{1.8} \mathrm{Ba}_{0.2} \mathrm{Ca}_{2} \mathrm{Cu}_{3} \mathrm{O}_{x}$ superconductor is shown in Fig. 4. Two regions within the superconducting transition were selected for the analysis, giving the exponents $\lambda_{1}=0.23 \pm 0.05$ in the temperature range [117.96 K, $118.74 \mathrm{~K}]$ and $\lambda_{2}=2.12 \pm 0.03$ in the temperature range $[118.74 \mathrm{~K}, 123.56 \mathrm{~K}]$. In the interval where the critical fluctuations are observed, the full dynamical scaling theory [32] predicts that the excess conductivity expressed by (2) diverges at the critical temperature with the critical exponent given by the formula

$\lambda=v(2+z-d+\eta)$

where $v$ is the critical exponent for the coherence length, $z$ is the dynamical exponent, $d$ is the dimensionality of the fluctuations spectrum, and $\eta$ is the exponent for the order 


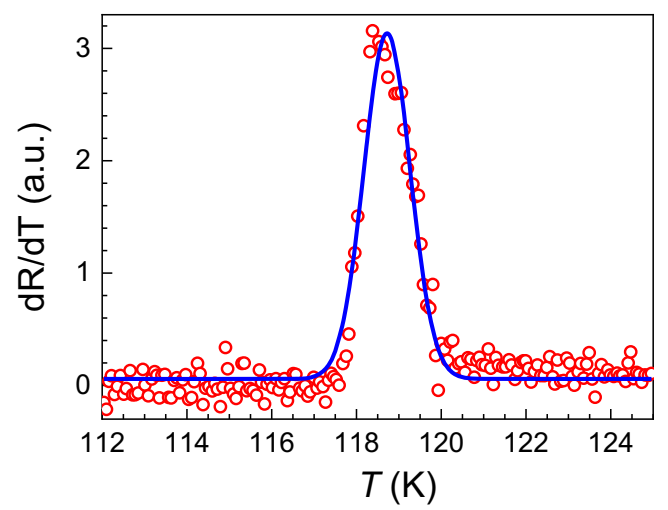

Fig. 3 Temperature dependence of the first derivative of the resistance of the $\mathrm{Tl}_{0.8} \mathrm{Bi}_{0.3} \mathrm{Sr}_{1.8} \mathrm{Ba}_{0.2} \mathrm{Ca}_{2} \mathrm{Cu}_{3} \mathrm{O}_{x}$ (1223) bulk superconductor and its fit with a single Gaussian function

parameter of the correlation function. The thermodynamic properties of superconductors in the critical region are the same as predicted for the 3D-XY model. Following the paper [33], one can substitute $v=2 / 3, z=3 / 2$, and $\eta=0$. Then, taking into account the calculated values of the critical exponents, we can determine the dimensionality of critical fluctuations. This dimensionality $\left(d_{\mathrm{c}}\right)$ in the critical region is

$d_{\mathrm{c}}=(7-3 \lambda) / 2$

As mentioned above, in the temperature region above the critical temperature, the critical exponents are usually dominated by stochastic (Gaussian) fluctuations. The Ginzburg-Landau mean field theory predicts that $v=1 / 3$, $z=2$, and $\eta=0$. Thus, following the paper [21, 22], the dimensionality $\left(d_{\mathrm{g}}\right)$ of stochastic (Gaussian) fluctuations can be described as follows

$d_{\mathrm{g}}=4-3 \lambda$

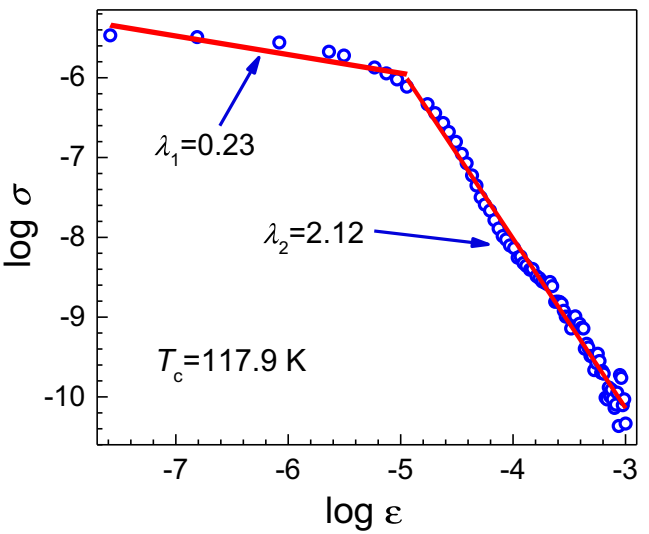

Fig. 4 Dependence of $\log \Delta \sigma$ versus $\log \varepsilon$ for bulk $\mathrm{Tl}_{0.8} \mathrm{Bi}_{0.3} \mathrm{Sr}_{1.8} \mathrm{Ba}_{0.2} \mathrm{Ca}_{2} \mathrm{Cu}_{3} \mathrm{O}_{x}$ with straight lines fitted to the linear regions giving $\lambda_{1}$ and $\lambda_{2}$ critical exponents. The zero critical temperature $T_{\mathrm{c} 0}=117.9 \mathrm{~K}$ was used to derive $\varepsilon$
For the first region, the closest to the zero resistance critical temperature $T_{\mathrm{c} 0}$, we find that the value of critical exponent $\lambda_{1}$ is 0.23 . Assuming that this region is dominated by critical fluctuations $[19,20]$, using (7), we evaluated the dimensionality of the fluctuations in the first region at $d_{\mathrm{c} 1}=3.16$. This result is in a good agreement with the assumption of 3D-XY universality class for our case. The second critical exponent $\lambda_{2}=2.12$ is too large to be considered and analyzed within this theory. To compare the effect of critical temperature versus the argument $\varepsilon$ on the values of critical exponents, a log-log calculation was made for the critical temperature at which the maximum $\mathrm{d} R / \mathrm{d} T$ is observed. The graph illustrating (5), in which the argument $\varepsilon$ contains the critical temperature $T_{\mathrm{cp}}=118.7 \mathrm{~K}$ that has been set as the position of the peak of $\mathrm{d} R / \mathrm{d} T$, is shown in Fig. 5. As previously, the two regions of the superconducting transition were selected for the analysis, giving the respective exponents: $\lambda_{1}=0.22 \pm 0.05$ in the temperature range $[118.74 \mathrm{~K}, 119.12 \mathrm{~K}]$ and $\lambda_{2}=$ $1.24 \pm 0.02$ in the temperature range $[119.20 \mathrm{~K}, 121.94 \mathrm{~K}]$. The critical exponents $\lambda_{1}$ in the region close to the critical temperature are virtually the same. However, $\lambda_{2}$ decreases from 2.12 to 1.24 when the critical temperature increases from 117.8 to $118.7 \mathrm{~K}$. While critical exponent 2.12 cannot be interpreted within the present phase transition theories, the value of 1.24 indicates that, according to the theory described in papers $[21,22,28]$, we can deal with fluctuations between $2 \mathrm{D}$ and $1 \mathrm{D}$ case.

At the second stage, the critical exponents were determined from the a.c. susceptibility data. These data were analyzed within a simple approach in which the fluctuations of the magnetic susceptibility are described within a power law that can be written in the following way:

$\chi=C \varepsilon^{-\lambda}$,

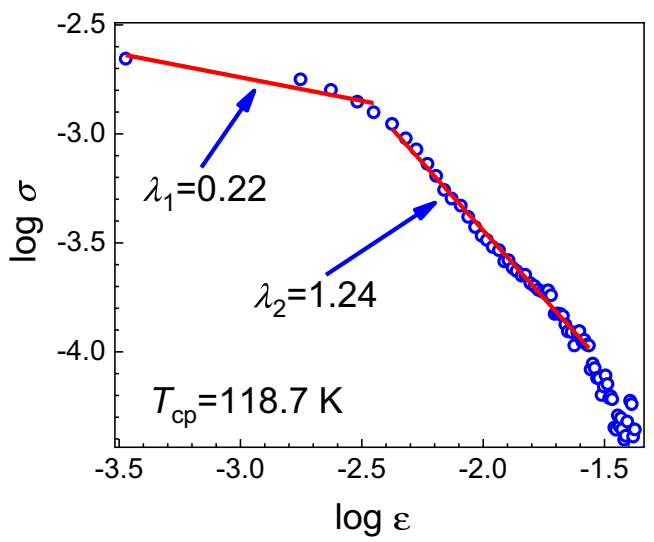

Fig. 5 Dependence of $\log \Delta \sigma$ versus $\log \varepsilon$ for bulk $\mathrm{Tl}_{0.8} \mathrm{Bi}_{0.3} \mathrm{Sr}_{1.8} \mathrm{Ba}_{0.2} \mathrm{Ca}_{2} \mathrm{Cu}_{3} \mathrm{O}_{x}$ sample with straight lines fitted to the linear regions giving $\lambda_{1}$ and $\lambda_{2}$ critical exponents with the critical temperature $T_{\mathrm{cp}}=118.7 \mathrm{~K}$ used to derive $\varepsilon$ 

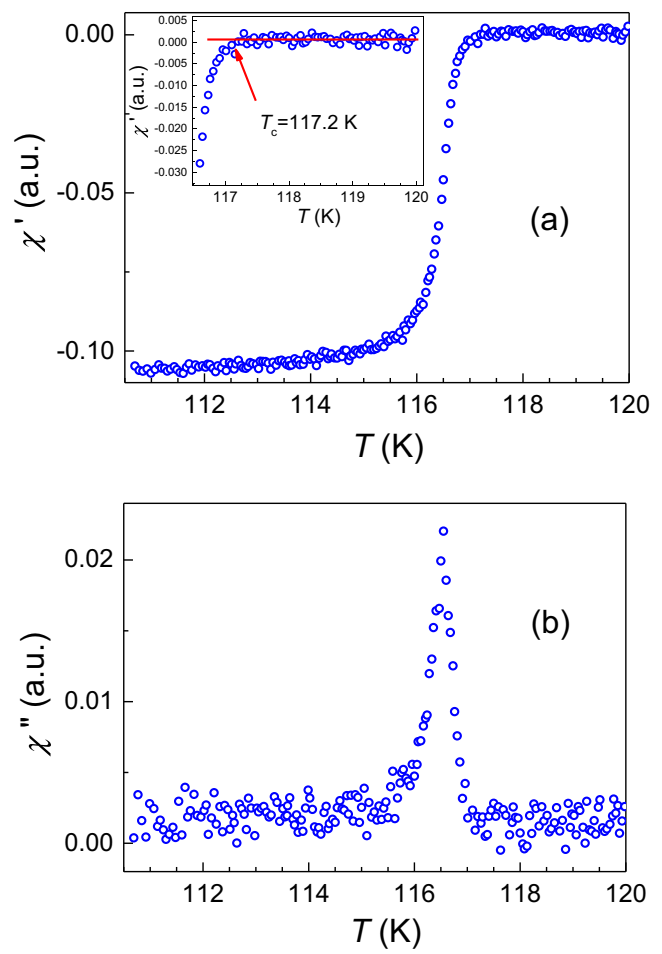

Fig. 6 Dispersion (a) and absorption (b) components of a.c susceptibility of $\mathrm{Tl}_{0.8} \mathrm{Bi}_{0.3} \mathrm{Sr}_{1.8} \mathrm{Ba}_{0.2} \mathrm{Ca}_{2} \mathrm{Cu}_{3} \mathrm{O}_{x}$ superconductor measured at the a.c. magnetic field amplitude of $2 \mathrm{mOe}$. Inset: the inter-grain critical temperature $T_{\mathrm{c}}=117.2 \mathrm{~K}$ obtained from the point in which the non-zereo $\chi^{\prime}$ appears

where $\varepsilon=\left|\frac{T-T_{c}}{T_{c}}\right|, T_{\mathrm{c}}$ is the critical temperature, $\lambda$ is the critical exponent, and $C$ is a constant.

The a.c. susceptibility can be written as a complex number by the formula

$\chi=\chi^{\prime}+i \chi^{\prime \prime}$,

where $\chi^{\prime}$ is the dispersion and $\chi^{\prime \prime}$ is the absorption part of the a.c. susceptibility and $i^{2}=-1$.

Experimental data for these components of the susceptibility are shown in Fig. 6a, b, respectively. The measurements were done at the a.c. magnetic field amplitude of 20 mOe. One can notice a small width of the transition to the superconducting state which is consistent with the result obtained from the resistance vs. temperature measurement. The inter-grain critical temperature $T_{\mathrm{c}}=117.2 \mathrm{~K}$ is taken from the point in which the non-zero $\chi^{\prime}$ appears (see the inset of Fig. 6a). In the analysis according to (9), the modulus of the a.c. susceptibility is defined as:

$\bmod (\chi)=\sqrt{\left(\chi^{\prime}\right)^{2}+\left(\chi^{\prime \prime}\right)^{2}}$,

The modulus of the measured components of a.c. susceptibility is presented in Fig. 7 as a function of temperature.

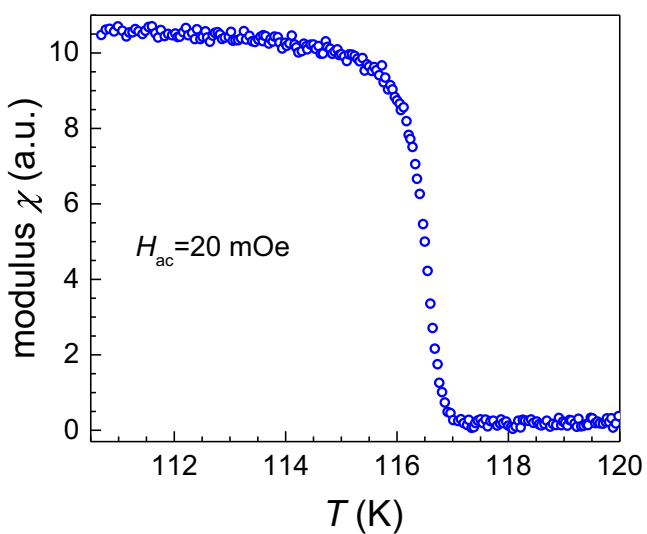

Fig. 7 Modulus of the parts of the a.c. susceptibility measured as a function of temperature

The critical exponent $\lambda$ is derived from (9) which can be converted to the following formula

$\log (\bmod \chi)=-\lambda \log \varepsilon+$ const,

where the critical exponent $\lambda$, defined as previously, is equal to the value of the slope of the line fitted to the linear parts of this dependence.

The relation obtained from (12) for the $\mathrm{Tl}_{0.8} \mathrm{Bi}_{0.3} \mathrm{Sr}_{1.8} \mathrm{Ba}_{0.2}$ $\mathrm{Ca}_{2} \mathrm{Cu}_{3} \mathrm{O}_{x}$ superconductor is shown in Fig. 8. For the analysis, two regions within the superconducting transition were selected and they give the critical exponents $\lambda_{3}=$ $0.30 \pm 0.18$ for the temperature range $[116.91 \mathrm{~K}, 117.15 \mathrm{~K}]$ and $\lambda_{4}=2.48 \pm 0.07$ for the temperature range $[116.33 \mathrm{~K}$, $116.91 \mathrm{~K}]$. The zero critical temperature $T_{\mathrm{c} 0}=117.2 \mathrm{~K}$ was used to derive $\varepsilon$. In the vicinity of the critical temperature, the critical fluctuations also show threedimensional character, $d_{\mathrm{c} 1}=3.05$, which is in a good agreement with the $3 \mathrm{D}-\mathrm{XY}$ universality class. It is worth noting that the critical exponents derived close to the

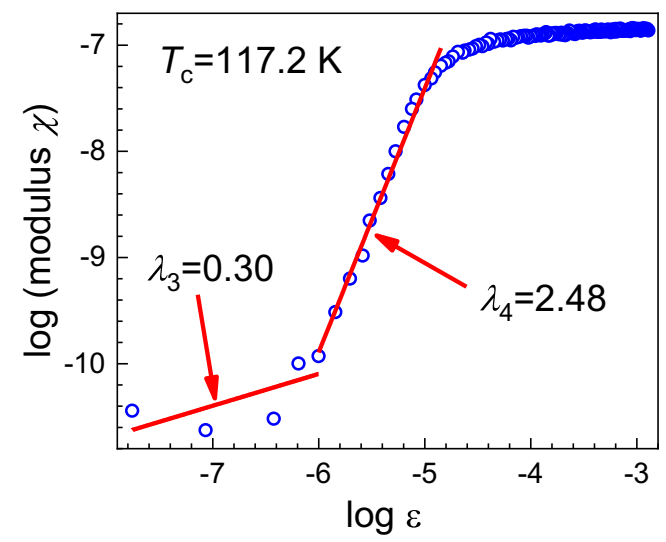

Fig. $8 \log ($ modulus $(\chi))$ versus $\log (\varepsilon)$ for the bulk $\mathrm{Tl}_{0.8} \mathrm{Bi}_{0.3} \mathrm{Sr}_{1.8} \mathrm{Ba}_{0.2} \mathrm{Ca}_{2} \mathrm{Cu}_{3} \mathrm{O}_{x}$ with straight lines fitted to the linear regions providing $\lambda_{3}$ and $\lambda_{4}$ critical exponents. The zero critical temperature $T_{\mathrm{c} 0}=117.2 \mathrm{~K}$ was used to derive $\varepsilon$ 
Table 1 Critical exponents obtained both from the conductivity and the susceptibility data together with the temperature ranges in which they were derived from
Critical exponents

Temperature range (K)

\begin{tabular}{lll}
\hline From conductivity data for $T_{\mathrm{c} 0}=117.9 \mathrm{~K}$ & & \\
$\lambda_{1}$ & $0.23 \pm 0.05$ & $(117.96,118.74)$ \\
$\lambda_{2}$ & $2.12 \pm 0.03$ & $(118.74,123.56)$ \\
From conductivity data for $T_{\mathrm{cp}}=118.7 \mathrm{~K}$ & & \\
$\lambda_{1}$ & $0.22 \pm 0.05$ & $(118.74 \mathrm{~K}, 119.12 \mathrm{~K})$ \\
$\lambda_{2}$ & $1.24 \pm 0.02$ & $(119.20 \mathrm{~K}, 121.94 \mathrm{~K})$ \\
From susceptibility data for $T_{\mathrm{c}}=117.2 \mathrm{~K}$ & & \\
$\lambda_{3}$ & $0.30 \pm 0.18$ & $(116.91,117.15)$ \\
$\lambda_{4}$ & $2.48 \pm 0.07$ & $(116.33,116.91)$
\end{tabular}

critical temperature from both the susceptibility (slightly below $T_{\mathrm{c}}$ ) as well as the resistance (slightly above $T_{\mathrm{c}}$ ) measurements are close to each other: $\lambda_{3}=0.30$ and $\lambda_{1}=0.23$ respectively. All the obtained critical exponent values are collected in Table 1 . They show that in the bulk $\mathrm{Tl}_{0.8} \mathrm{Bi}_{0.3} \mathrm{Sr}_{1.8} \mathrm{Ba}_{0.2} \mathrm{Ca}_{2} \mathrm{Cu}_{3} \mathrm{O}_{x}$ superconductor, three-dimensional behavior of superconductivity around the critical temperature occurs. Surprisingly, the critical exponents derived from the region farther from the critical temperature, both below and above it, have comparable values: $\lambda_{4}=2.48$ and $\lambda_{2}=2.12$ respectively. When the temperature decreases, the resistance decreases due to new percolation paths formed through the weak links (Josephson junctions). Therefore, in this region, the fluctuations with the Cooper pairs that flow through the intergranular area are concerned. Below the critical temperature, the susceptibility signal is mainly related to the grain interiors, so the respective Cooper pair fluctuations are related to the intragranular regions. As described in several papers [5, 27, $34,35]$, the critical exponents bigger than 1.5 do not find reliable theoretical explanations. In several works [36-38], one can find suggestions that such high values of critical exponents are related to the properties of the Josephson junction. It means that the granular structure of the HTS is the most likely responsible for the large spectrum of their parameters observed.

\section{Summary and Conclusions}

The results of this work can be summarized as follows:

1. The temperature dependencies measured for the electrical conductivity and the a.c. susceptibility enabled us to study the transition from normal to the superconducting state for the $\mathrm{Tl}_{0.8} \mathrm{Bi}_{0.3} \mathrm{Sr}_{1.8} \mathrm{Ba}_{0.2} \mathrm{Ca}_{2} \mathrm{Cu}_{3} \mathrm{O}_{x}$ bulk superconductor with the critical temperature $T_{\mathrm{c}, 50 \%}=118.8 \mathrm{~K}$ and the width of superconducting transition $\Delta T=1.6 \mathrm{~K}$.
2. In the electrical conductivity data, the two regions within the superconducting transition are distinguished, giving the exponents $\lambda_{1}=0.23 \pm 0.05$ and $\lambda_{2}=$ $2.12 \pm 0.03$. For the first region, the closest to the zero critical temperature $T_{\mathrm{c} 0}$, the critical exponent $\lambda_{1}$ value reveals the three-dimensional character of the fluctuations. The second critical exponent $\lambda=2.12$ is too large to be analyzed and explained within the present theories. If the temperature at which the $\mathrm{d} R / \mathrm{d} T$ reaches the maximum is set as the critical temperature, the following values of the critical exponents are obtained: $\lambda_{1}=0.22 \pm 0.05$ and $\lambda_{2}=1.24 \pm 0.02$. The critical exponents $\lambda_{1}$ in the region close to the critical temperature are nearly the same. However, $\lambda_{2}$ decreases from 2.12 to 1.24 when the critical temperature increases from 117.8 to $118.7 \mathrm{~K}$. The value of 1.24 can be interpreted within the theory described in papers [21, 22, 29] and can possibly be related to fluctuations between $2 \mathrm{D}$ and $1 \mathrm{D}$ cases

3. In the a.c. magnetic susceptibility data also two regions within the superconducting transition were distinguished, giving the critical exponents: $\lambda_{3}=$ $0.30 \pm 0.18$ and $\lambda_{4}=2.48 \pm 0.07$. In the vicinity of the critical temperature, the critical fluctuations with the exponent $\lambda_{3}$ show also three-dimensional character, which is in a good agreement with the $3 \mathrm{D}-\mathrm{XY}$ universality class.

4. The critical exponents derived from the region close to the critical temperature for both the susceptibility (slightly below $T_{\mathrm{c}}$ ) as well as the electrical resistance (slightly above $T_{\mathrm{c}}$ ) data are close to each other: $\lambda_{3}=$ 0.30 and $\lambda_{1}=0.23$ respectively.

5. The large values of the critical exponents in the region farther from the critical temperature, like $\lambda_{4}=2.48$ and $\lambda_{2}=2.12$, obtained in the region farther from the critical temperatures are not explainable within current theories. However, their values depend on the choice of critical temperature and, for $T_{\mathrm{cp}}=118.7 \mathrm{~K}$, the 
critical exponent obtained, $\lambda_{2}=1.24$, can be related to fluctuations between $2 \mathrm{D}$ and $1 \mathrm{D}$ cases.

Acknowledgments This work was supported by the AGH UST statutory tasks No. 11.11.220.01/6 within a subsidy of the Ministry of Science and Higher Education. The authors would like to thank Professor Czesław Kapusta for his valuable comments on the manuscript.

Open Access This article is distributed under the terms of the Creative Commons Attribution 4.0 International License (http:// creativecommons.org/licenses/by/4.0/), which permits unrestricted use, distribution, and reproduction in any medium, provided you give appropriate credit to the original author(s) and the source, provide a link to the Creative Commons license, and indicate if changes were made.

\section{References}

1. Ma, Z., Taber, R.C., Lombardo, L.W., Kapitulnik, A., Beasley, M.R., Merchant, P., Eom, C.B., Hou, S.Y., Phillips, J.M.: Phys. Rev. Lett. 71, 781 (1993)

2. see for instance: Narlikar A.V. (ed.) (2004) High Temperature Superconductivity 1, Materials. Springer, Berlin

3. Woch, W.M., Kowalik, M., Giebułtowski, M., Zalecki, R., Szeliga, A., Przewoźnik, J., Kapusta, Cz.: J. Supercond. Nov. Magn. 30, 569 (2017)

4. Woch, W.M., Chrobak, M., Kowalik, M., Zalecki, R., Przewoźnik, J., Kapusta Cz.: J. Supercond. Nov. Magn. 29, 2333 (2016)

5. Freitas, P.P., Tsuei, C.C., Plaskett, T.S.: Phys. Rev. B 36, 833 (1987)

6. Ausloos, M., Laurent, Ch.: Phys. Rev. B 37, 611 (1988)

7. Vidalt, F., Veirat, J.A., Mazat, J., Garcia-Alvarado, F., Moran, E., Alario, M.A.: J. Phys. C: Solid State Phys. 21, L599 (1988)

8. Balestrino, G., Nigro, A., Vaglio, R., Marinelli, M.: Phys. Rev. B 39, $12246(1989)$

9. Lee, C., Cho, J.H., Johnston, D.C.: Phys. Rev. B 43, 457 (1991)

10. Kim, D.H., Gray, K.E., Kampwirth, R.T., McKay, D.M.: Phys. Rev. B 43, 2910 (1991)

11. Khan, N.A., Ashraf, M.N., Safeer, S.H.: J. Supercond. Nov. Magn. 29, 2253 (2016)

12. Ranjbar, M.G., Abd-Shukor, R.: J. Supercond. Nov. Magn. 29, 2235 (2016)
13. Pureur, P., Menegotto Costa, R., Rodrigues, P., Schaf, J. Jr., Kunzler, J.V.: Phys. Rev. B 47, 11420 (1993)

14. Menegotto Costa, R., Pureur, P., Gusmao, M., Senoussi, S., Behnia, K.: Phys. Rev. B 64, 214513 (2001)

15. Mendoca Ferreira, L., Pureur, P., Borges, H.A., Lejay, P.: Phys. Rev. B 69, 212505 (2004)

16. Woch, W.M., Chrobak, M., Kowalik, M., Zalecki, R., Giebułtowski, M., Niewolski, J., Gondek, Ł.: J. All. Comp. 692, 359 (2017)

17. see for instance: Job, B., Rosenberg, M., Physica C, 172, 391 (1991) and references therein

18. Rietveld, H.M.: J. Appl. Crystallogr. 2, 65 (1969)

19. Woch, W.M., Zalecki, R., Kołodziejczyk, A., Sudra, H., Gritzner, G.: Supercond. Sci. Technol. 21, 085002 (2008)

20. Sudra, H., Gritzner, G., Horhager, N., Weber, H.W.: Supercond. Sci. Technol. 21, 075010 (2008)

21. Aslamazov, L.G., Larkin, A.I.: Sov. Phys. Solid State 10, 875 (1968)

22. Larkin, A.I., Varlamov, A.A.: In: Bennemann K.H., Ketterson J.B. (eds.) Superconductivity, vol. 1, p. 369. Springer, Berlin (2008)

23. Kim, D.H., Gray, K.E., Trochet, M.D.: Phys. Rev. B 45, 10801 (1992)

24. Duan, H.M., Kiehl, W., Dong, C., Cordes, A.W., Saeed, M.J., Viar, D.L., Hermann, A.M.: Phys. Rev. B 43, 12925 (1991)

25. Woch, W.M., Chrobak, M., Kołodziejczyk, A.: Acta Phys. Pol. A 118, 389 (2010)

26. Ahmad, N.H., Khan, N.A., Yahya, A.K.: J. Alloys Compd. 492, 473 (2010). and references therein

27. Han, S.H., Axnas, J., Zhao, B.R., Rapp, O.: Physica C 408-410, 679 (2004)

28. Skocpol, W.J., Tinkham, M.: Rep. Prog. Phys. 38, 1409 (1975)

29. Ginzburg, V.I., Landau, L.D., Eksperim, Zh.: Teor. Fiz. 20, 1064 (1950)

30. Mohanta, A., Behera, D.: Physica C 470, 295 (2010)

31. Solovjov, A., Tkachenko, M., Vovk, R., Chroneos, A.: Physica C 501, 24 (2014)

32. Hohenberg, F.C., Halperin, B.I.: Rev. Mod. Phys. 49, 435 (1977)

33. Lobb, C.J.: Phys. Rev. B 36, 3930 (1987)

34. Menegotto Costa, R., Rieel, I.C., Jurelo, A.R., Pimentel, J.L.: J. Mag. Magn. Mater. 320, 493 (2008)

35. Woch, W.M., Chrobak, M., Kołodziejczyk, A., Gritzner, G.: Acta Phys. Pol., A 121, 1059 (2012)

36. Grusniko, M.A., Mors, P.M.: Phys. Rev., B 42, 10030 (1990)

37. Fischer, D.S., Fisher, M.P.A., House, D.: Phys. Rev., B 43, 130 (1991)

38. Lebeau, C., Rosenblatt, J., Raboutou, A., Peyral, P.: Europhys. Lett. 1, 313 (1986) 